TRANSACTIONS OF THE

AMERICAN MATHEMATICAL SOCIETY

Volume 348, Number 8, August 1996

\title{
TOPOLOGICAL ENTROPY OF STANDARD TYPE MONOTONE TWIST MAPS
}

\author{
OLIVER KNILL
}

\begin{abstract}
We study invariant measures of families of monotone twist maps $S_{\gamma}(q, p)=\left(2 q-p+\gamma \cdot V^{\prime}(q), q\right)$ with periodic Morse potential $V$. We prove that there exist a constant $C=C(V)$ such that the topological entropy satisfies $h_{\text {top }}\left(S_{\gamma}\right) \geq \log (C \cdot \gamma) / 3$. In particular, $h_{\text {top }}\left(S_{\gamma}\right) \rightarrow \infty$ for $|\gamma| \rightarrow \infty$. We show also that there exist arbitrary large $\gamma$ such that $S_{\gamma}$ has nonuniformly hyperbolic invariant measures $\mu_{\gamma}$ with positive metric entropy. For large $\gamma$, the measures $\mu_{\gamma}$ are hyperbolic and, for a class of potentials which includes $V(q)=\sin (q)$, the Lyapunov exponent of the map $S$ with invariant measure $\mu_{\gamma}$ grows monotonically with $\gamma$.
\end{abstract}

\section{INTRODUCTION}

For a one-parameter family of smooth diffeomorphisms $S_{\gamma}$ on a two-dimensional compact manifold $M$, the topological entropy $h_{t o p}\left(S_{\gamma}\right)$ depends continuously on $\gamma$, and there exists for each $\gamma$ a $T_{\gamma}$-invariant ergodic measure of maximal entropy [New 89]. Moreover, according to Katok [Kat 80] (see also [You 81]), the map $S_{\gamma}$ is a limit of hyperbolic subshifts of finite type: if $h_{t o p}\left(S_{\gamma}\right)>0$, there exist $S_{\gamma}$-invariant hyperbolic sets having entropy arbitrarily close to $h_{t o p}\left(S_{\gamma}\right)$. It follows that a change of the topological entropy is accompanied by creations or destructions of hyperbolic sets. Any of these sets $K_{\gamma}$ is the support of an invariant measure $\mu_{\gamma}$ having metric entropy equal to the topological entropy of $S_{\gamma}$ restricted to $K_{\gamma}$. A general hyperbolic measure $\mu_{\gamma}$ depends continuously on the parameter $\gamma$. The abstract dynamical system, $\left(Y, S_{\gamma}, \mu_{\gamma}\right)$ is an ergodic subshift of finite type embedded in the topological dynamical system $\left(Y, S_{\gamma}\right)$. At the boundary of a maximal interval for which this Markov chain is embedded, there still exists an invariant measure $\mu_{\gamma}$ but $\left(S_{\gamma}, \mu_{\gamma}\right)$ is in general only a measure theoretical factor of $(S, T, m)$, in which case we say it is an immersion.

In this paper, we study embeddings and immersions of dynamical systems in generalized standard maps $S_{\gamma}:(q, p) \mapsto\left(q^{\prime}, p^{\prime}\right)=\left(2 q-p+\gamma \cdot V^{\prime}(q), q\right)$ on the torus $Y=\mathbf{T}^{2}$. Such maps are discrete Hamiltonian systems and have a natural parametrization by the coupling constant $\gamma$. The twist property allows one to describe $S_{\gamma}$ by variational methods. Immersions of a given dynamical system

Received by the editors July 25, 1994.

1991 Mathematics Subject Classification. Primary 58F11, 28D20, 28D10; Secondary 58E30, $58 \mathrm{~F} 05$.

Key words and phrases. Ergodic theory, monotone twist maps, topological entropy, hyperbolic sets, Lyapunov exponents, invariant measures, variational principles.

This material is based upon work which was supported by the National Science Foundation under Grant No. DMS-9101715. The Government has certain rights in this material.

(C)1996 American Mathematical Society 
$(X, T, m)$ are in one to one correspondence with critical points of a class of variational functionals. For large $|\gamma|$, critical points can be found with the implicit function theorem. This idea is due to Aubry and Abramovici [Aub 90] and is called the "anti-integrable limit". Together with some ergodic theory this will show that $h_{\text {top }}\left(S_{\gamma}\right) \rightarrow \infty$ for $|\gamma| \rightarrow \infty$. We stress that we get here quantitative results about the topological entropy, namely a lower bound of the form $a+b \log (\gamma)$ for some constants $a, b$. The existence of homoclinic points or other arguments like [Ang 90, Ang 92] give qualitative results.

For a fixed standard map $S_{\gamma}$, all the possible variational functionals on $L^{\infty}(X)$ are labeled by measurable integer valued functions $N \in L^{\infty}(X, 2 \pi \mathbf{Z})$. They fall into equivalence classes, and the quotient space is a cohomology space $H(T)$ attached to $(X, T, m)$. The number $\int N d m$ is a function on $H(T)$. To each embedded system belongs an element of the cohomology space. It follows that if two embedded systems belong to different elements $H(T)$, then they are different. The numerical invariant $\int N d m$ can be used to get multiplicity results, i.e. to get uncountably many different critical points.

There are other invariants of the embedding, such as the integrated density of states of the Hessian at a critical point. This invariant is up to a constant the Morse index in the case when the variational problem is finite dimensional, i.e. if a periodic orbit is embedded.

With an implicit function theorem approach similar to the one for finding critical points for large $|\gamma|$, one can compute the stable and unstable direction-fields on the hyperbolic set. These direction-fields are tangent to the stable and unstable manifolds and related to the Titchmarsh-Weyl functions of the Hessian, a discrete ergodic Schrödinger operator. By computing the parameter dependence of the critical point belonging to a hyperbolic invariant measure $\mu_{\gamma}$ for large $|\gamma|$, we will show that these stable and unstable direction fields depend monotonically on $\gamma$. It follows that the Lyapunov exponent of $S_{\gamma}$ is growing monotonically for large $|\gamma|$. This, combined with a theorem of Fathi, tells us that there is an explicit upper bound on the Hausdorff dimension of the hyperbolic set which is monotonically decreasing as $|\gamma| \rightarrow \infty$.

Proving compactness of the immersions, we will show also that for arbitrary large $\gamma$, there exist invariant measures with positive Lyapunov exponents but which do not have a hyperbolic set as support. It is an open question if such sets form a set of positive Lebesgue measure for large $\gamma$.

At the end of the article, we show how some of the ideas can be carried over to higher dimensional symplectic maps, real or complex Hénon like maps. We will also treat noninvertible systems like one-dimensional circle maps, complex polynomial or meromorphic maps. The Aubry-Abramovici perturbation idea applies also to discrete partial difference equations such as, for example, coupled map lattices, where it gives embedded two-dimensional shifts.

\section{INVARIANT MEASURES OF MONOTONE TWIST MAPS}

2.1. Immersions and embeddings of dynamical systems. A measure theoretical dynamical system $(X, T, m)$ is a measure preserving invertible map $T$ on a Lebesgue probability space $(X, m)$. A topological dynamical system $(Y, S)$ is a homeomorphism $S$ on a compact topological space $Y$. 
Definition 2.1. A measure theoretical dynamical system $(X, T, m)$ is embedded in a topological dynamical system $(Y, S)$, if there exists an $S$-invariant probability measure $\mu$ on $Y$, such that $(X, T, m)$ and $(Y, S, \mu)$ are isomorphic as measure theoretical dynamical systems.

Definition 2.2. If the system $(Y, S, \mu)$ is only a factor of $(X, T, m)$ we speak of an immersion. Two embeddings or immersions are called different, if the measures are different.

A basic question is to decide whether and how often a given abstract dynamical system $(X, T, m)$ can be embedded or immersed. Given two embeddings of the same dynamical system, one cannot use purely ergodic theoretical invariants to distinguish the embeddings because both embedded systems are isomorphic. Other invariants should allow one to distinguish different embeddings, and hopefully label the set $\mathcal{E}_{T}(S)$ of embeddings and the set $\mathcal{I}_{T}(S)$ of immersions.

According to Goodman's variational theorem, the metric entropy $h_{m}(T)$ of an embedded dynamical system must be bounded from above by the topological entropy $h_{\text {top }}(S)$. This necessary condition for an embedding is in general not sufficient: $h_{m}(T) \leq h_{t o p}(S)$ does not in general allow an embedding. An example is a uniquely ergodic topological dynamical system $(Y, S)$ with $h_{t o p}(S)>0$, because obviously no system $(X, T, m)$ with metric entropy smaller than $h_{t o p}(S)$ can be embedded.

2.2. The ergodic Percival functional. We study the embedding problem in the case when the topological dynamical system $(Y, S)$ is a monotone twist map. Embedding an ergodic dynamical system $(X, T, m)$ is then a variational problem. We consider the special case of generalized standard maps

$$
S:(q, p) \mapsto\left(q^{\prime}, p^{\prime}\right)=\left(2 q-p+V^{\prime}(q), q\right),
$$

with $V \in C^{2}\left(\mathbf{T}^{1}, \mathbf{R}\right)$. The map $S$ acts also on the torus $Y=\mathbf{T}^{2}$, because $S(q, p+2 \pi)=\left(q^{\prime}, p^{\prime}+2 \pi\right)$ and the topological entropy $h_{\text {top }}(S)$ is defined. Consider for each $N \in L^{\infty}(X, 2 \pi \mathbf{Z})$ the ergodic Percival functional

$$
q \mapsto \mathcal{L}_{N}(q)=\int_{X}[h(q(x), q(T x))-N(x) q(x)] d m(x)
$$

on the Banach space $L^{\infty}(X, \mathbf{R})$, where $h\left(q, q^{\prime}\right)=-\left(q^{\prime}-q\right)^{2} / 2-V(q)$ is the generating function of the twist map. These functionals $\mathcal{L}_{N}$ are bounded and have the same smoothness as $V$. Define $\Delta(q)=q(T)-2 q+q\left(T^{-1}\right)$.

Lemma 2.3. If there exists $q \in L^{\infty}(X)$ satisfying the Euler equations

$$
\delta \mathcal{L}_{N}(q)=\Delta(q)-V^{\prime}(q)-N=0,
$$

then $(X, T, m)$ is immersed in the twist map $(Y, S)$.

Proof. The homomorphism between $(X, T, m)$ and the embedded system $(Y, S, \mu)$ is given by the map $\phi: X \rightarrow Y$,

$$
x \mapsto\left(q(x), q\left(T^{-1} x\right)\right) \bmod 2 \pi \mathbf{Z} .
$$

The condition $S \circ \Phi=\Phi \circ T$ follows immediately from $\delta \mathcal{L}_{N}(q)=0$. The pushforward measure $\mu$ on $Y$ defined by $\mu(Z)=m\left(\phi^{-1}(Z)\right)$ is $S$-invariant.

If $q$ is not constant, then the immersed factor is nontrivial in the sense that the immersed system has not only fixed points. If $\phi$ is injective almost everywhere on $X$, the system $(X, T, m)$ itself is embedded. 
Remark 2.4. The fact that we look at bounded measurable functions $q$ forces the introduction of a family of variational functionals. The case $N=0$ gives only a few embeddings.

Remark 2.5. Most of the literature about monotone twist maps deals with a variational problem in a space of sequences.

Other versions of the functional $\mathcal{L}$ have been introduced earlier. For the embedding of irrational rotations, we refer to [Per 80], [Mat 82], [Laz 84]. The ergodic functional $\mathcal{L}$ for general dynamical systems $(X, T, m)$ appeared in [Kni 93] in the case $N=0$.

Example 2.6. Every embedded system corresponds to a critical point of a variational problem: given an $S$-invariant probability measure $m$ on $Y$, define the dynamical system $(\operatorname{supp}(m), S, m)$, where $T$ is the restriction of $S$ to the support of $m$. Choose a function $K \in L^{\infty}(X, 2 \pi \mathbf{Z})$. Denote by $\pi_{1}: \mathbf{T} \times \mathbf{R} \rightarrow \mathbf{T}$ the projection onto the first coordinate. The function $q(x)=\pi_{1}(x)+K(x)$ is a critical point of the functional $\mathcal{L}_{N}(q)$ with $N=\Delta(q)-V^{\prime}(q)$. The choice of the function $K$ determines the chosen lift from $q \in L^{\infty}\left(X, \mathbf{T}^{1}\right)$ to $L^{\infty}(X, \mathbf{R})$.

Example 2.7. An embedded finite ergodic dynamical system $T: x_{i} \mapsto x_{i+1}$ corresponds to periodic orbits. According to the Poincaré-Birkhoff theorem (see [Bro 75]), there exist many periodic orbits of each period.

Example 2.8. The embedding of irrational rotations $x \mapsto x+\alpha$ of the circle is well investigated. Functions $q(x)=x+v$ with smooth $v$ correspond to KAM tori (see for example [Mos 73], [Her 83]). If $v$ is discontinuous then $q$ belongs to an invariant Cantor set such as the Aubry-Mather sets (see [Mat 82, Kat 82, Ban 88]).

Example 2.9. Immersions of subshifts of finite type are possible, if there exist homoclinic points (see for example [Fon 90]). Embeddings of Bernoulli shifts can be obtained by constructing horseshoes. If a Bernoulli shift $\left(I^{\mathbf{Z}}, T, m\right)$ is embedded, Krieger's theorem (see for example [Den 76]) assures that every ergodic dynamical system with metric entropy $\leq \log (|I|)$ can be embedded.

\section{INVARIANTS OF EMBEDDINGS}

The Hessian at a critical point $q$ of $\mathcal{L}_{N}$ is the Fréchet derivative of

$$
\delta \mathcal{L}: L^{\infty}(X) \rightarrow L^{\infty}(X), \quad q \mapsto \Delta(q)-V^{\prime}(q)
$$

and is a discrete Schrödinger operator on $L^{\infty}(X)$ defined by

$$
L u=\Delta u-V^{\prime \prime}(q) u .
$$

One can extend this operator to a bounded linear operator on $L^{2}(X)$. For $m$-almost all $x \in X$, we get a family of ergodic Jacobi matrices $L(x): l^{2}(\mathbf{Z}) \rightarrow l^{2}(\mathbf{Z})$ defined by

$$
(L(x) u)_{n}=u_{n+1}-2 u_{n}+u_{n-1}+V^{\prime \prime}(q(x)) u_{n} .
$$

Attached to such a measurable family of operators is the density of states $d k$ (see for example [Cyc 87]). This is a measure on the real line having as its support in the ergodic case the spectrum $\sigma(L(x))$ for almost all $x \in X$.

Definition 3.1. A critical point $q$ is called hyperbolic, if its Hessian $L$ is invertible. For ergodic $(X, T, m)$, this is equivalent to $0 \notin \sigma(L(x))$ for almost all $x \in X$. 
The embedded system of a hyperbolic critical point is a hyperbolic set (see [Aub 92a]). Therefore, we call an invariant measure defined by a hyperbolic critical point also hyperbolic, and say also the embedding is hyperbolic if $\mu$ is hyperbolic. If $\mu$ is a hyperbolic invariant measure, then it defines by the implicit function theorem a family of measures $S^{\prime} \mapsto \mu_{S^{\prime}}$, for $S^{\prime}$ in a neighborhood $S$. A simple example of a hyperbolic set is a hyperbolic critical point of a finite dynamical system, which is a hyperbolic periodic orbit.

Definition 3.2. A stable invariant of an embedding is a map $\psi$ from the set $\mathcal{E}_{T}(S)$ into some topological space so that for any hyperbolic embedding $\mu, S^{\prime} \mapsto \psi\left(\mu_{S^{\prime}}\right)$ is constant for $S^{\prime}$ in a neighborhood of $S$.

Example 3.3. An example of a stable invariant is the topological index of a periodic orbit. It is useful for classifying and counting periodic orbits. Another example of a stable invariant is the Morse index of a periodic orbit, if a periodic orbit can be characterized as a critical point of some functional.

One motivation for considering invariants of embeddings is that it allows us to distinguish different invariant measures $\mu, \nu \in \mathcal{E}_{T}(S)$ which are not distinguishable from the ergodic point of view.

The density of states $d k(\mu)$, the spectrum $\sigma(\mu)$ of $L$ and the integrated density of states are functions on $\mathcal{M}_{S}$, but not stable invariants of the embedding, since a general perturbation of $S$ can change them even if $\mu$ is hyperbolic.

\subsection{The generalized Morse index.}

Definition 3.4. The generalised Morse index $k$ of an embedding is defined as $k(\mu):=\int_{-\infty}^{0} d k(E)$, where $d k$ is the density of states of the ergodic operator $L$. (The measure $d k$ is defined via the Riesz representation theorem by the functional $f \mapsto \int f(L(x))_{00} d m(x)$ on $C(\mathbf{R})$.)

Proposition 3.5. The generalized Morse index is a stable invariant of the embedding.

Proof. If $\mu$ is hyperbolic, the Hessian is invertible and 0 is not in the spectrum. This stays true for $S^{\prime}$ in a neighborhood of $S$. The value of $k$ therefore does not change under perturbations of $S$.

For a hyperbolic periodic orbit, the classical Morse index $\tilde{k}$ is related to the generalised Morse index by $k=\tilde{k} / Q$, where $Q$ is the period of the orbit [Mat 84].

\subsection{A cohomology set.}

Definition 3.6. Call two functions $N_{1}, N_{2} \in L^{\infty}(X, 2 \pi \mathbf{Z})$ T-cohomologous, $N_{1} \sim_{T} N_{2}$, if there exist $K \in L^{\infty}(X, 2 \pi \mathbf{Z})$ and an automorphism $U$ commuting with $T$ such that $N_{1}-N_{2}(U)=\Delta K$. Define the cohomology set

$$
H(T)=L^{\infty}(X, 2 \pi \mathbf{Z}) / \sim_{T} .
$$

Denote by $\mathcal{M}_{S}$ the set of $S$-invariant probability measures and let $\mathcal{E}_{S}(T) \subset \mathcal{M}_{S}$ be the set of $S$-invariant probability measures which are embeddings of $(X, T, m)$.

Lemma 3.7. The function $\Psi(\mu):=\left(\Delta(q)-V^{\prime}(q)\right) / \sim_{T}$ maps $\mathcal{E}_{S}(T)$ into $H(T)$ and is independent of the critical point $q$ belonging to $\mu \in \mathcal{E}_{S}(T)$. 
Proof. Let $q_{1}, q_{2}$ be two critical points with the same measure $\mu$ and let

$$
\phi_{i}:(X, T, m) \rightarrow(Y, S, \mu), \quad x \mapsto\left(q_{i}(x), q_{i}\left(T^{-1} x\right)\right)
$$

be the corresponding conjugations. The automorphism $U: X \rightarrow X, x \mapsto \phi_{1}^{-1} \phi_{2}(x)$ satisfies $T U=U T$ and $\phi_{1}(U)=\phi_{2}$. From the first coordinate of the equation $\phi_{1}(U)=\phi_{2}$, we read off $q_{1}(U)-q_{2}=K \in L^{\infty}(X, 2 \pi \mathbf{Z})$, which means

$$
N_{2}=\Delta\left(q_{2}\right)-V^{\prime}\left(q_{2}\right)=\Delta\left(q_{1}(U)\right)-V^{\prime}\left(q_{1}(U)\right)+\Delta K=N_{1}(U)+\Delta K
$$

and $N_{2} \sim_{T} N_{1}$.

Proposition 3.8. $\psi$ is a stable invariant of the embedding.

Proof. Attached to every $\mu \in \mathcal{I}_{S}(T)$ is a critical point of the functional $\mathcal{L}_{N}$, and $\psi(\mu)$ is given by $\psi(\mu)=N / \sim_{T}$. Assume $\mu$ is hyperbolic. Let $q_{S}$ be the critical point corresponding to $\mu_{S}$. By the implicit function theorem, there exists a critical point $q_{S^{\prime}}$ of $\mathcal{L}_{N}$ for $S^{\prime}$ near $S$, and $\psi\left(\mu_{S}\right)=\psi(\mu)$, as long as $q_{S}$ is a critical point of $\mathcal{L}_{N}$.

\subsection{The average force.}

Definition 3.9. The trace $\rho: L^{\infty}(X, 2 \pi \mathbf{Z}) \rightarrow \mathbf{R}, N \mapsto \int N d m$ projects to a function $\rho: H(T) \rightarrow \mathbf{R}$ which we call the average force. Define $\rho$ on $\mathcal{E}_{S}(T)$ by

$$
\rho(\mu)=\rho \circ \Psi(\mu) .
$$

Because $\int \Delta(q) d m(x)=0$, we can rewrite $\rho$ with the Euler equation $\Delta(q)=$ $V^{\prime}(q)+N$ as

$$
\rho(\mu)=-\int V^{\prime}(q(x)) d m(x),
$$

where $q$ is any critical point belonging to the measure $\mu$. This explains the name average force.

Corollary 3.10. The average force $\rho$ is a stable invariant of the embedding.

Proof. This follows from Proposition 3.8 and $\rho(\mu)=\int \psi(\mu) d m$.

Because it is difficult to decide whether two functions $N, N^{\prime}$ are $T$-cohomologous or not, the simpler invariant $\rho$ is useful and leads to multiplicity results.

\section{Hyperbolic inVARIANT MEASURES AND THE TOPOLOGICAL ENTROPy}

Denote by $\Sigma \subset \mathbf{T}$ the set of nondegenerate critical points of the Morse function $V \in C^{2}(\mathbf{T})$. We consider a one-parameter family of twist maps $S_{\gamma}$ on $Y=\mathbf{T}^{2}$ given by

$$
S_{\gamma}:(q, p) \mapsto\left(2 q-p+\gamma \cdot V^{\prime}(q), q\right) .
$$

Given $q_{0} \in L^{\infty}(X, \Sigma+2 \pi \mathbf{Z})$, denote by $q_{0}(X)$ the finite set $\left\{\sigma \in \mathbf{R} \mid m\left(q_{0}^{-1}(\sigma)\right)>\right.$ $0\}$.

Theorem 4.1. Fix $N \in L^{\infty}(X, 2 \pi \mathbf{Z})$ and $q_{0} \in L^{\infty}(X, \Sigma+2 \pi \mathbf{Z})$ and an ergodic dynamical system $(X, T, m)$ with metric entropy $h_{m}(T) \leq \log \left(\left|q_{0}(X)\right|\right)$. There exists $\gamma_{0}=\gamma\left(N, q_{0}\right)>0$ such that for $|\gamma|>\gamma_{0}$, the system $(X, T, m)$ can be embedded. That is, there exist hyperbolic measures in $\mathcal{E}_{S_{\gamma}}(T)$ with metric entropy $\log \left(\left|q_{0}(X)\right|\right)$. 
Proof. By Krieger's theorem, it is enough to embed a Bernoulli shift $(X, T, m)=$ $\left(q_{0}(X)^{\mathbf{Z}}, T, m\right)$ with entropy $\log \left|q_{0}(X)\right|$. In this case, any system $(X, T, m)$ with smaller entropy can be embedded.

Define $\epsilon=1 / \gamma$. The variational functional $\epsilon \cdot \mathcal{L}_{N}(q)$ has the Euler equations

$$
\epsilon \cdot \Delta(q)=V^{\prime}(q)+\epsilon N q
$$

For $\epsilon=0$, every $q \in L^{\infty}(X, \Sigma)$ is a critical point. Because $V$ is a Morse function, the Hessian $L=-V^{\prime \prime}(q)$ of such a critical point is invertible and the implicit function theorem implies the existence of the critical point also for small $\epsilon<\epsilon_{N}$. The map $\phi(x)=\left(q(x), q\left(T^{-1}\right)\right)$ conjugates $(X, T, m)$ with $(Y, S)$, and $(X, T, m)$ is an immersion. The map $\phi$ is injective: if $\phi(x)=\phi(y)$, then $\phi\left(T^{n} x\right)=\phi\left(T^{n} y\right)$ and comparing the first coordinates of $\phi\left(T^{n} x\right)=\phi\left(T^{n} y\right)$ gives $x_{n}=y_{n}$, and so $x=y$.

The twist map

$$
S:(q, p) \mapsto\left(2 q-p+\gamma \cdot V^{\prime}(q), q\right)
$$

on $Y=\mathbf{R}^{2} /(2 \pi \mathbf{Z})^{2}$ is conjugated by $(q, p) \mapsto(q / k, p / k)$ to the twist map

$$
S_{k}:(q, p) \mapsto\left(2 q-p+\frac{\gamma}{k} \cdot V^{\prime}(k \cdot q), q\right)
$$

on the torus $Y_{k}=\mathbf{R}^{2} /((2 \pi / k) \cdot \mathbf{Z})^{2}$. The torus $Y$ is a $k^{2}$-sheeted cover of $Y_{k}$, and we can lift $S_{k}$ to a map $\tilde{S}_{k}$ on $Y$. The potential $\tilde{V}(x)=V(k x)$ has $k \cdot|\Sigma|$ different critical points. For large $\gamma$, we can embed a shift $(X, T, m)$ with $k \cdot|\Sigma|$ symbols by a map $\tilde{\phi}$. Denote by $\tilde{\mu}$ the invariant measure given by this embedding. The system $\left(Y, \tilde{S}_{k}, \tilde{\mu}\right)$ has metric entropy $\log (k \cdot|\Sigma|)$. The projection $\pi: Y \mapsto Y_{k}$ satisfies $\pi \circ \tilde{S}_{k}=S_{k} \circ \pi$. Call $\mu=\pi^{*} \tilde{\mu}$ the push-forward of $\tilde{\mu}$ by the projection $\pi$. Because $\left(Y, \tilde{S}_{k}, \tilde{\mu}\right)$ is a finite skew-product extension of $\left(Y, S_{k}, \mu\right)$, we know from the Abramov-Rokhlin formula (see for example [Pet 83]) that the metric entropy of $\left(Y_{k}, S_{k}, \mu\right)$ is the same as the metric entropy of $\left(Y, \tilde{S}_{k}, \tilde{\mu}\right)$. Using Ornstein's results that a factor of a Bernoulli shift is again a Bernoulli shift [Orn 70] and that the isomorphy class of Bernoulli shifts is determined by the metric entropy, we have embedded by $\phi=\pi \circ \tilde{\phi}$ the Bernoulli shift with entropy $\log (k \cdot|\Sigma|)$, and not only a factor.

Remark 4.2. The idea to go to the limit $|\gamma| \rightarrow \infty$ is due to Aubry [Aub 90] and is called anti-integrable limit. The idea has been used further in [Aub 92a, Aub 92b].

Remark 4.3. If $\left|q_{0}(X) \bmod (2 \pi \mathbf{Z})\right| \geq 2$ and the metric entropy of $(X, T, m)$ is small enough, then $\mathcal{E}_{S_{\gamma}}(T)$ is not empty.

Remark 4.4. Given $k \in[0,1]$ and $N \in H(T)$, if the dynamical system $(X, T, m)$ is aperiodic, there exists an immersion with Morse index $k$. We only have to choose the function $q_{0}$ such that $m\left\{x \in X \mid V^{\prime \prime}\left(q_{0}(x)\right)<0\right\}=k$. If $h_{m}(T)>0$ and $q_{0}$ is not constant, the immersion is not trivial.

Remark 4.5. Critical points $q, q^{\prime}$ with the same average force and Morse index need still not belong to the same embedding: for a fixed cohomology class $N$, there are $2^{Q}$ periodic orbits for each of these periodic orbits. There are $Q ! /(k !)(Q-k) !$ orbits with the same Morse index $k$.

Proposition 4.6. Using the notation of Theorem 4.1, we can choose $\gamma\left(N, q_{0}\right)$ satisfying

$$
\gamma\left(N, q_{0}\right)=3 \cdot \max \left\{\left|V^{\prime \prime \prime}\right|, 4\right\} \cdot\left(\max \left\{\left|\Delta q_{0}-N\right|_{\infty},|| L_{0}^{-1}||\right\}\right)^{3} .
$$


Proof. Differentiating the Euler equation $\epsilon \cdot \Delta(q)+V^{\prime}(q)=\epsilon \cdot N$ with respect to $\epsilon$ and denoting the derivative with respect to $\epsilon$ with a dot gives

$$
\dot{q}=-L^{-1}(\Delta(q)-N) .
$$

With $\dot{L}=V^{\prime \prime \prime}(q) \cdot \dot{q}$ we have

$$
\begin{aligned}
\frac{d}{d \epsilon}\left\|L^{-1}\right\| & \leq\left\|\frac{d}{d \epsilon} L^{-1}\right\| \leq\left\|L^{-1}\right\|^{2} \cdot\|\dot{L}\| \\
& \leq\left\|L^{-1}\right\|^{3} \cdot\left|V^{\prime \prime \prime}\right|_{\infty} \cdot|\Delta q-N|_{\infty} .
\end{aligned}
$$

Also

$$
\frac{d}{d \epsilon}|\Delta q-N|_{\infty} \leq 4 \cdot|\dot{q}|_{\infty} \leq 4 \cdot|| L^{-1}|| \cdot|\Delta q-N|_{\infty} .
$$

These two differential inequalities can be written with $a=\left\|L^{-1}\right\|$ and $b=$ $|\Delta q-N|_{\infty}$ as

$$
\dot{a} \leq\left|V^{\prime \prime \prime}\right|_{\infty} b a^{3}, \dot{b} \leq 4 b a .
$$

The function $c=\max \{a, b, 1\}$ satisfies

$$
\dot{c} \leq \max \left\{\left|V^{\prime \prime \prime}\right|_{\infty}, 4\right\} \cdot c^{4} .
$$

Therefore, a solution $L^{-1}(\epsilon)$ exists for

$$
\epsilon \leq\left(3 \cdot \max \left\{\left|V^{\prime \prime \prime}\right|, 4\right\} \cdot\left(\max \left\{\left|\Delta q_{0}-N\right|_{\infty},|| L_{0}^{-1}||\right\}\right)^{3}\right)^{-1} .
$$

Corollary 4.7. For any fixed Morse function $V \in C^{3}\left(\mathbf{T}^{1}\right)$, there exists a constant $C$ such that $h_{\text {top }}\left(S_{\gamma}\right) \geq \log (C \gamma) / 3$ for all $\gamma$.

Proof. Proposition 4.6 implies that $\gamma\left(N, q_{0}^{(n)}\right) \leq M \cdot n^{3}$ with some constant $M$. Take a sequence of functions $q_{0}^{(n)} \in L^{\infty}(X, 2 \pi \mathbf{Z})$ with $\left|q_{0}^{(n)}(X)\right|=n$. For $\gamma>$ $M \cdot n^{3} \geq \gamma\left(N, q_{0}^{(n)}\right)$, a shift with $n$ symbols can be embedded and therefore

$$
h_{\text {top }}\left(S_{\gamma}\right) \geq \log (n) \text {. }
$$

Corollary 4.8. Given a Morse function $V \in C^{3}\left(\mathbf{T}^{1}\right)$, let $(X, T, m)$ be a dynamical system of finite entropy. For $\gamma$ large enough, there exist simultaneous embeddings such that the average force takes any value in $[0,1]$.

Proof. The average force $\int_{X} N d m$ can take any value in $[0,1]$ for $N$ in $L^{\infty}(X,\{0,2 \pi\})$. Take $\gamma$ larger than a lower bound given in Proposition 4.6 for all $N \in L^{\infty}(X,\{0,2 \pi\})$.

\section{IMMERSED SYSTEMS}

We defined $\mathcal{E}_{S}(T)$ as the set of all $S$-invariant probability measures $\mu$ such that $(X, T, m)$ and $(Y, S, \mu)$ are isomorphic. This set is in general not closed in the compact set $\mathcal{M}_{S}$ of all $S$-invariant probability measures, as the following simple example shows. Take $X=\mathbf{T}^{1}, T(x)=x+\alpha$ with $\alpha$ irrational and $S(z)=e^{i \alpha} z$ on $Y=\{|z| \leq 1\}$. The sequence $\mu_{n} \in \mathcal{E}_{S}(T)$ of Lebesgue measures on $\{|z|=1 / n\}$ converges to the invariant measure $\mu$ on the fixed point 0 , which is no more in $\mathcal{E}_{S}(T)$. We obtain compactness, however, if we consider the set $\mathcal{I}_{S}(T)$ of all $S$ invariant probability measures $\mu$ on $Y$ such that $(Y, S, \mu)$ is a factor of $(X, T, m)$ : 
Lemma 5.1. Given an abstract dynamical system $(X, T, m)$ and a topological $d y$ namical system $(Y, T)$, where $Y$ is a compact topological space. The set $\mathcal{I}_{S}(T)$ is compact in $\mathcal{M}_{S}$. Moreover, if $S_{n} \rightarrow S$ in the uniform topology $C(Y, Y)$ and $\mu_{n} \in \mathcal{I}_{S_{n}}(T)$, then $\mu_{n}$ has an accumulation point in $\mathcal{I}_{S}(T)$.

Proof. Assume $\mu_{n} \in \mathcal{I}_{S_{n}}(T)$ converges to $\mu \in \mathcal{M}_{S}$. There exists a $T$-invariant set $X^{\prime}$ of full measure and a sequence of measurable maps $\phi_{n}: X \rightarrow Y \subset \mathbf{R}^{2}$ such that $\phi_{n} T(x)=S \phi_{n}(x)$ for all $x \in X^{\prime} \subset X$ and all $n>0$. The sequence $\phi_{n}$ has by Tychonov an accumulation point $\phi$ in the space $Y^{X^{\prime}}$ of all functions $X^{\prime} \rightarrow Y$. Pass to a converging subsequence. The function $\phi$ is measurable as a pointwise limit of measurable functions. We have

$$
\phi_{n}(T x) \rightarrow \phi(T x), \quad x \in X^{\prime},
$$

since $\phi_{n}$ converges pointwise and $X^{\prime}$ is $T$-invariant. Since $S$ is continuous and $S_{n} \rightarrow S$ in $C(Y, Y)$, we obtain

$$
S_{n} \phi_{n}(x) \rightarrow S \phi(x), \quad x \in X^{\prime} .
$$

(1) and (2) together imply that $\phi T(x)=S \phi(x)$ pointwise for all $x \in X^{\prime}$. Because $\mu_{n} \rightarrow \mu$ and by Lebesgue's dominated convergence theorem, we have for all $f \in$ $C(Y)$

Therefore

$$
0=\int f d \mu_{n}-\int f\left(\phi_{n}\right) d m \rightarrow \int f d \mu-\int f(\phi) d m .
$$

$$
\int_{X} f(\phi(x)) d m(x)=\int_{Y} f(y) d \mu(y), \quad \forall f \in C(Y) .
$$

This shows that $\phi$ is measure preserving as a map from $(X, m)$ to $(Y, \mu)$.

Remark 5.2. In the twist map case, if $\mu_{n} \in \mathcal{I}_{S}(T) \rightarrow \mu \in \mathcal{I}_{S}(T)$, there exists a sequence of critical points $q_{n}$ which converges pointwise to a critical point $q$ with the measure $\mu$. The set of critical points of $\mathcal{L}_{N}$ in $L^{\infty}(X)$ is therefore compact in the topology of pointwise convergence.

Definition 5.3. An $S_{\gamma}$-invariant measure $\mu_{\gamma}$ is called nonuniform hyperbolic, if it is not hyperbolic but its Lyapunov exponent is positive.

\subsection{Nonuniform hyperbolicity.}

Proposition 5.4. For all $\gamma>0$, there exists $\gamma^{\prime}>\gamma$ such that $S_{\gamma^{\prime}}$ has a nonuniform hyperbolic invariant measure.

Proof. Let $(X, T, m)$ be a Bernoulli shift with finite entropy and choose $N$ with $\int_{X} N d m \notin 2 \pi \mathbf{Z}$. The function $N$ is not $T$-cohomologous to a constant function, and every measure $\nu \in \mathcal{I}_{S}(T)$ is aperiodic since $T^{n}$ is ergodic. For large $\gamma$, hyperbolic measures $\mu_{\gamma} \in \mathcal{E}_{S}(T)$ exist which embed the shift. Take $\gamma^{\prime}=\inf \left\{\gamma \mid \mu_{\gamma}\right.$ hyperbolic $\}$. This gives by the compactness lemma 5.1 a measure $\mu_{\gamma^{\prime}}$ which is aperiodic. By Ruelle's formula (see for example [Man 87]), the Lyapunov exponent satisfies $\lambda\left(\mu_{\gamma}\right) \geq$ $h_{\mu_{\gamma}}(T)>0$. On the other hand, $\mu_{\gamma}$ is not hyperbolic.

Remark 5.5. For all $\gamma>0$, there exists $\gamma^{\prime}>\gamma$ such that $S_{\gamma^{\prime}}$ has a parabolic fixed point. Proof. Given $\gamma>0$, choose $N \in 2 \pi \mathbf{Z}$ so large that no solution $q$ of the equation $\gamma \cdot V^{\prime}(q)=N$ exists. For $\gamma^{\prime \prime}>\gamma$ large enough, there exists a solution. Choose $\gamma^{\prime}$ as the infimum over all values for which there exists a hyperbolic solution. The fixed point for $S_{\gamma^{\prime}}$ is parabolic. 
Remark 5.6. For all $\gamma>0$, there exists $\gamma^{\prime}>\gamma$ such that $S_{\gamma^{\prime}}$ has parabolic periodic orbits of period 2. Proof. Fix $\gamma=1 / \epsilon$. Take $|X|=\{1,2\}$ and write $q(i)=q_{i}$. Choose $N_{1}=N(1)=0$ and $N_{2}=N(2)$ with $N_{2} / \gamma>2\left\|V^{\prime}\right\|_{\infty}$. Adding the two equations

$$
2 \epsilon\left(q_{1}-q_{2}\right)-V^{\prime}\left(q_{2}\right)=0, \quad 2 \epsilon\left(q_{2}-q_{1}\right)-V^{\prime}\left(q_{1}\right)=\epsilon N_{2},
$$

we obtain $V^{\prime}\left(q_{1}\right)+V^{\prime}\left(q_{2}\right)=\epsilon N_{2}$, which has no solution because we assumed $N_{2} \epsilon>2\left\|V^{\prime}\right\|_{\infty}$. If we take $\epsilon^{\prime \prime}=1 / \gamma^{\prime \prime}$ small enough, we get a hyperbolic critical point. Therefore, there exists a value $\gamma^{\prime}$, between $\gamma$ and $\gamma^{\prime \prime}$, where the hyperbolic periodic orbit becomes parabolic. Since there are no critical points with $q_{1}=q_{2}$, the parabolic orbit really has period two.

\section{Asymptotic Behaviour of the Critical points for Large $\gamma$}

Proposition 6.1. Fix $N$ and $(X, T, m)$. For "time" $\epsilon=1 / \gamma=0$, many hyperbolic critical points of $\mathcal{L}_{N}$ appear instantaneously. Each of these critical points $q$ satisfies the differential equation $\dot{q}=-L^{-1}(q)(\Delta q-N)$. The critical points are labeled by the initial velocity $\dot{q}_{0}=-L^{-1}\left(q_{0}\right)\left(\Delta q_{0}-N\right)$.

Proof. The motion of a hyperbolic critical point $q=q_{\epsilon}$ is given for $\epsilon=0$ by $\dot{q}=-L^{-1} V^{\prime}(q) / \epsilon=-L^{-1}(q)(\Delta q-N)$. In the limiting case $\gamma=\infty$, we still have $\dot{q}=-L^{-1}\left(q_{0}\right)\left(\Delta q_{0}-N\right)$, which depends on $q_{0}$ and $N$. For $\gamma=\infty$, many hyperbolic critical points are born if we consider $\epsilon=1 / \gamma$ as time. These critical points can be labeled by the values of $\left(\Delta q_{0}-N\right)$ by the uniqueness of solutions of differential equations in Banach spaces.

Remark 6.2. Solving the differential equation of the critical points allows us to compute hyperbolic periodic orbits of $S_{\gamma}$ numerically for large $\gamma$.

Proposition 6.3. Fix $N \in L^{\infty}(X, 2 \pi \mathbf{Z})$ and $q_{0} \in L^{\infty}(X, \Sigma+2 \pi \mathbf{Z})$. Denote by $q_{\epsilon}$ a critical point of $\mathcal{L}_{N}$ (satisfying $q_{\epsilon} \rightarrow q_{0}$ for $\epsilon \rightarrow 0$ ), and by $\mu_{\epsilon}$ the corresponding hyperbolic invariant measure for $\gamma=1 / \epsilon$. If $|\epsilon|$ is small enough, then $\operatorname{sign}\left(\dot{q}_{\epsilon}\right)=$ $\operatorname{sign}\left(V^{\prime}\left(q_{\epsilon}\right) V^{\prime \prime}\left(q_{\epsilon}\right)\right)$. For every $x \in X$, the value $q_{\epsilon}(x)$ moves monotonically away from $\Sigma+2 \pi \mathbf{Z}$ as $\epsilon$ is increasing.

Proof. Differentiation of the Euler equations $\epsilon(\Delta(q)-N)=V^{\prime}(q)$ with respect to $\epsilon$ gives

$$
\left(\epsilon \Delta-V^{\prime \prime}(q)\right) \dot{q}=-(\Delta(q)-N)=-V^{\prime}(q) / \epsilon .
$$

Dividing both sides by $V^{\prime \prime}(q)$ leads to

$$
\dot{q}=\left(1-\frac{\epsilon \Delta}{V^{\prime \prime}(q)}\right)^{-1} \frac{V^{\prime}(q)}{V^{\prime \prime}(q)}=(1+O(\epsilon)) \frac{V^{\prime}(q)}{V^{\prime \prime}(q) \epsilon} .
$$

Therefore, for small $\epsilon$,

$$
\operatorname{sign}(\dot{q}(x))=\operatorname{sign}\left(\frac{V^{\prime}(q(x))}{V^{\prime \prime}(q(x))}\right)=\operatorname{sign}\left(V^{\prime}(q(x)) \cdot V^{\prime \prime}(q(x))\right) .
$$

Because

$$
\frac{d}{d \epsilon}\left(V^{\prime}(q(x))\right)^{2}=V^{\prime}(q(x)) V^{\prime \prime}(q(x)) \dot{q}(x)>0
$$

for all $x$, the map $\epsilon \mapsto\left|V^{\prime}\left(q_{\epsilon(x)}\right)\right|$ is monotonically increasing, which means that $q_{\epsilon}(x)$ is moving away from the critical set $\Sigma+2 \pi \mathbf{Z}$ of $V$. 
Lemma 6.4. Let $(X, T, m)$ be any dynamical system. Given a one parameter family $a_{t}$ of functions in $L^{\infty}(X)$ satisfying $\left|a_{t}(x)\right| \geq\left|a_{s}(x)\right|$ for $t \geq s$. If $\left|b_{t}\right|_{\infty}=$ $\left|1 / a_{t}\right|_{\infty}$ is small enough, the Lyapunov exponent

$$
\lambda\left(A_{t}\right)=\lim _{n \rightarrow \infty} \frac{1}{n} \int_{X} \log \left\|A_{t}\left(T^{n-1}(x)\right) \cdots A_{t}(x)\right\| d m(x)
$$

of $x \mapsto A_{t}(x)=\left(\begin{array}{cc}a_{t}(x) & -1 \\ 1 & 0\end{array}\right)$ is monotonically increasing in $t$.

Proof. If $|1 / a|_{\infty}$ is small enough, the discrete Schrödinger operator $\left(L_{a}(x) u\right)_{n}=$ $u_{n+1}+u_{n-1}+a\left(T^{n} x\right) u_{n}$ is invertible. There exist two solutions $u^{ \pm} \in R^{\mathbf{Z}}$ of $L_{a}(x) u=0$ which are in $l^{2}( \pm \mathbf{N})$. The Titchmarsh-Weyl functions $m_{a}^{ \pm}(x)=$ $u_{n+1}^{ \pm} / u_{n}^{ \pm}$are real (possibly infinite), and the Lyapunov exponent satisfies $\lambda\left(A_{a}\right)=$ $\int_{X} \log \left(m_{a}^{-}(x)\right) d m(x)$. The function $m_{a}^{-}$satisfies the discrete Ricatti equation $m_{a}^{-}(T)+a+1 / m_{a}^{-}=0$. For $b=1 / a$ and $l=1 / m^{-}$, this is equivalent to

$$
F(b, l)=b+l(T)+b \cdot l \cdot l(T)=0 .
$$

Because $F(0,0)=0$ and $D_{2} F(0,0) u=u(T)$, the implicit function theorem implies for small $|b|_{\infty}$ a solution $G \in L^{\infty}(X)$ of $F(b, G(b))=0$ with $m^{-}(a)=1 / G(1 / a)$. From $D F(b, G(b))=0$, we get, writing again $l=G(b)$,

$$
1+D l+l \cdot l(T)+b(D l \cdot l(T)+l \cdot D l(T))=0,
$$

which shows that $D l=-1+O(\|b\|)$. It follows that $b \mapsto 1 / l(b)$ is monotone for small $|b|_{\infty}$. Therefore, also the map

$$
b \mapsto \lambda(b)=\int_{X} \log \left(\frac{1}{l(b(x))}\right) d m(x)
$$

is monotone as well as $t \mapsto \lambda\left(A_{t}\right)$.

The Lyapunov exponent of a critical point $q$ is defined as the Lyapunov exponent

$$
\lambda(\gamma)=\lim _{n \rightarrow \infty} \frac{1}{n} \int_{\mathbf{T} \times \mathbf{T}} \log \left\|d\left(S_{\gamma}^{n}\right)\right\| d \mu_{\gamma}
$$

of $S_{\gamma}$ with respect to the invariant measure $\mu_{\gamma}$ belonging to $q_{\gamma}$.

Corollary 6.5. Assume $V^{\prime \prime}$ is a Morse function containing as critical points also the critical points of $V($ i.e. $V(q)=\sin (q))$. Then, for small enough $\epsilon$, the Lyapunov exponent of $\mu_{\epsilon}$ increases monotonically as $\epsilon \rightarrow 0$.

Proof. The transfer matrix

$$
x \mapsto A_{\epsilon}(x)=\left(\begin{array}{cc}
\frac{1}{\epsilon} \cdot V^{\prime \prime}(q(x))+2 & -1 \\
1 & 0
\end{array}\right) \in S L(2, \mathbf{R})
$$

of $L_{\epsilon}$ is the Jacobean $d S_{\gamma}$ of the twist map with $\gamma=1 / \epsilon$. The assumption on the potential $V$ implies that $V^{\prime \prime}(q)$ has local extrema at critical points of $V$. Proposition 6.3 together with the assumption on the potential implies that for large enough $\gamma$ and for all $x \in X$, the map $\gamma \mapsto\left|\gamma \cdot V^{\prime \prime}\left(q_{\gamma}(x)\right)\right|$ is monotone. Apply Lemma 6.4.

Remark 6.6. The Hausdorff dimension of the hyperbolic set $\operatorname{supp}\left(\mu_{\gamma}\right)$ goes to zero for $|\gamma| \rightarrow \infty$ since the Hausdorff dimension of the hyperbolic set is, by a theorem of Fathi [Fat 89], bounded above by $2 h_{t o p}\left(q_{\gamma}\right) / \lambda(\gamma)$, where $\lambda(\gamma)$ is the contraction rate of the uniform hyperbolic cocycle $D T(\gamma)$. It follows that the measure of the 
hyperbolic sets is zero for large $|\gamma|$. For hyperbolic sets which are locally maximal, it has been observed in [Aub 92a], using a result of Ruelle and Bowen [Bow 75], that the measure is zero.

Remark 6.7. If $V$ is real analytic, the supports of two different measures $\mu_{N}, \mu_{N^{\prime}}$ have in general a nonempty intersection, because there are only countably many different periodic orbits of a fixed period and the support of each $\mu_{N}$ contains many periodic orbits.

\section{Generalisations and Relations with other Systems}

7.1. Symplectic twist maps. A part of the above discussion can be generalized in a straightforward way to symplectic twist maps with generating function $l\left(q, q^{\prime}\right)=$ $-\left(q-q^{\prime}, q-q^{\prime}\right) / 2-\gamma \cdot V(q)$, where $V \in C^{2}\left(\mathbf{T}^{d}, \mathbf{R}\right)$. It is a map on $Y=\mathbf{T}^{2 d}$ given by $S:(q, p) \mapsto(2 q-p+\nabla V(q), q)$. An example of such a symplectic map is the three parameter Fröschle family with $V(q)=\gamma_{1} \cos \left(x_{1}\right)+\gamma_{2} \cos \left(x_{2}\right)+\mu \cos \left(x_{1}+x_{2}\right)$. To each $S$-invariant measure $m$, there corresponds a function $q \in L^{\infty}\left(X, \mathbf{R}^{d}\right)$ which is a critical point of the functional $\mathcal{L}_{N}(q)=\int_{X} l(q, q(T))+(N, q) d m$, where $N \in$ $L^{\infty}\left(X, 2 \pi \mathbf{Z}^{d}\right)$. The vector $\int_{X} N(x) d m(x)$ is the average force vector. There is a map from the set of invariant probability measures to the cohomology space $L^{\infty}\left(X, 2 \pi \mathbf{Z}^{d}\right) / \sim_{T}$. The second variation of $\mathcal{L}_{N}$ is a random discrete Schrödinger operator on the strip $L=\tau-2+\tau^{*}-\gamma \cdot V^{\prime \prime}(q)$, where $V^{\prime \prime}(q(x))$ is the Hessian of $V$ at a point $q(x) \in \mathbf{T}^{d}$. The generalized Morse index is defined as before. The topological entropy of $S_{\gamma}$ diverges as in the one-dimensional case.

7.2. Twist maps on the real or complex plane. Consider an Hénon type conservative twist map on $\mathbf{R}^{2}$ or $\mathbf{C}^{2}$ given by $S_{\gamma}:(x, y) \mapsto\left(2 x-y+\gamma \cdot V^{\prime}(x), x\right)$, where $V \in C^{2}\left(\mathbf{R}^{2}\right)$ (resp. $C^{2}\left(\mathbf{C}^{2}\right)$ ) is a Morse function having at least 2 critical points. In the variational functional, $N$ must vanish. Given a dynamical system $(X, T, m)$. A critical point of the functional $\mathcal{L}(q)=\int_{X}-(q(T)-q)^{2} / 2+V(q) d m$ corresponds to an embedding of the dynamical system in the twist map. There is no cohomology because we are the plane and not on the cylinder. For large enough $\gamma$, every system embedded in the twist map with potential $\gamma \cdot V$ is embedded as a hyperbolic set. Actually, most (with respect to Lebesgue measure) of the orbits of these Hénon maps may escape to infinity and there is a set left invariant. The generalized Morse index of an embedded system can be defined as before. The topological entropy of $S_{\gamma}$ is constant $\log (|\Sigma|)$ for large enough $\gamma$ (compare [Fri 89]).

7.3. Circle maps. The embedding question also makes sense for a noninvertible dynamical system $(X, T, m)$. Circle maps of the form $x \mapsto x+\alpha+\gamma \cdot V(x)$ on $\mathbf{T}^{1}=$ $\mathbf{R} /(2 \pi \mathbf{Z})$, with parameters $\gamma$ and $\alpha$ and $V \in C^{2}\left(\mathbf{T}^{1}\right)$ are called generalised Arnold maps. The variational functional does not exist, unlike the case of twist maps, but we can look at solutions $q \in L^{\infty}(X)$ of the equation $F_{N}(q)=q(T)-q-\alpha-\gamma \cdot V(q)-$ $N=0$, with $N \in L^{\infty}(X, 2 \pi \mathbf{Z})$. Given a zero $q$ of $F$, we have an immersion of the dynamical system defined by $\phi(x)=q(x)$. The implicit function theorem implies that for sufficiently large $\gamma$, the system $(X, T, m)$ (for example a one-sided Bernoulli shift) is embedded. The cohomology set is defined as $H(T)=L^{\infty}(X, 2 \pi \mathbf{Z}) / \sim_{T}$, where $N \sim_{T} N^{\prime}$ if and only if there exists an isomorphism $U$ commuting with $S$ such that $N(S)-N^{\prime}=K(T)-K$ for some $K \in L^{\infty}(X, 2 \pi \mathbf{Z})$. The average force of an embedded system is $\rho(q)=\int N d m$. The topological entropy is diverging to $\infty$ for $\gamma \rightarrow \infty$. 
7.4. Polynomial maps. Consider a family of polynomial maps $\gamma \cdot P: \mathbf{C} \mapsto \mathbf{C}$. There is no variational setup any more, but we can look for functions $q \in L^{\infty}(X)$ which satisfy $F(q)=q(T)-\gamma \cdot P(q)=0$. The derivative of $F$ is an operator $\tau-\gamma \cdot P^{\prime}$. The limit $\gamma \rightarrow \infty$ corresponds to $F(q)=P(q)$. If $\Sigma$ is the set of nondegenerate zeros of $P$, every $q \in L^{\infty}(X, \Sigma)$ is a solution. For large $\gamma=1 / \epsilon$, there is by the implicit function theorem also a zero $q$ of $\epsilon \cdot q(T)-P(q)$. The embedded systems have their support in the Julia set of the map $z \mapsto \gamma \cdot P(z)$. The topological entropy is bounded from above by $\log (\operatorname{deg}(P))$.

7.5. Meromorphic maps. Consider two entire maps $f, g$ and a family of meromorphic maps $z \mapsto \gamma f(z) / g(z)$. Examples are rational maps $z \mapsto \gamma \cdot P(z) / Q(z)$, where $P$ and $Q$ are polynomials or $z \mapsto \gamma \cdot \tan (z)$. To embed $(X, T, m)$, we seek solutions $q \in L^{\infty}(X)$ of $g(q) \cdot q(T)-\gamma \cdot f(q)=0$. Denote by $\Sigma_{f}, \Sigma_{g}$ the sets of simple zeros of $f$ and $g$. By the implicit function theorem, we have, for small $|\gamma|$, solutions $q$ near $L^{\infty}\left(X, \Sigma_{g}\right)$, and for large $|\gamma|$ solutions $q$ near $L^{\infty}\left(X, \Sigma_{f}\right)$. For meromorphic maps with infinitely many zeros and poles, we can embed a one-sided Bernoulli shift over any finite alphabet $I \subset \Sigma_{f}$, if $|\gamma|$ is large, and a one-sided Bernoulli shift over any finite alphabet $J \subset \Sigma_{g}$, if $|\gamma|$ is small enough. The topological entropy is then growing to $\infty$ for $\gamma \rightarrow \infty$ and $\gamma \rightarrow 0$.

7.6. Coupled map lattices. A coupled map lattice is obtained by taking any not necessarily invertible dynamical system $f=\gamma V^{\prime}: M \mapsto M$, where $M=\mathbf{T}^{n}$, extending $f$ to $M^{\mathbf{Z}}$ by $f(x)_{n}=f\left(x_{n}\right)$, and considering the map $S$ on $M^{\mathbf{Z}}$ defined by $S x=\epsilon \cdot \Delta f(x)+f(x) \bmod (2 \pi \mathbf{Z})$. This is an infinite chain of coupled dynamical systems which is for $\epsilon=0$ a lattice of uncoupled noninteracting maps. Because the time-evolution $S$ commutes with space translation $(U x)_{n}=x_{n+1}$, one can consider the system as a dynamical system with time $\mathbf{Z} \times \mathbf{N}$. Assume the set of hyperbolic fixed points $\Sigma$ of $V$ contains at least two points. Take the measure theoretical dynamical system $\left(X=\Sigma^{\mathbf{Z} \times \mathbf{N}}, T \times R, m\right)$, where $m$ is the product measure on $X$ and $T$ resp. $R$ is the two-sided resp. one-sided shift. We say that such a dynamical system is embedded in the coupled map lattice, if there exists a measure $\mu$ on $M^{\mathbf{Z}}$ which is invariant under $S$ and $U$ such that the systems $(X, T \times R, m)$ and $\left(M^{\mathbf{Z}}, U \times S, \mu\right)$ are isomorphic as measure theoretical $\mathbf{Z} \times \mathbf{N}$ dynamical systems. In order to embed systems, we look for solutions $q \in L^{\infty}(X)$ satisfying $\tilde{F}(q)=q(R)-\epsilon \cdot \Delta_{T} f(q)-f(q)=0$. With $\epsilon^{\prime}=1 / \gamma$, this can be written as $F(q)=\epsilon^{\prime} \cdot q(R)-\epsilon \cdot \Delta_{T} V^{\prime}(q)-V^{\prime}(q)=0$. For $\epsilon=\epsilon^{\prime}=0$, every $q \in L^{\infty}(X, \Sigma)$ is a solution. The implicit function theorem allows us to extend this solution for small enough $|\epsilon|,\left|\epsilon^{\prime}\right|$. The map $\phi: X \mapsto M^{\mathbf{Z}}, x \mapsto\left\{V^{\prime}\left(q\left(T^{n} x\right)\right)\right\}_{n \in \mathbf{Z}}$ embeds the system $(X, T \times R, m)$ into the coupled map lattice. A concrete example of such a system is $M=\mathbf{T}^{1}, V(x)=\cos (x)$. For $\gamma$ large enough and $\epsilon$ small enough, there is a two-dimensional shift embedded.

7.7. Discrete partial difference equations. One can also consider the problem to embed a $\mathbf{Z}^{d}$ dynamical system $(X, T, m)$ with $T=\left(T_{1}, \ldots, T_{d}\right)$ into a partial difference equation $\Delta(u)=\gamma \cdot V^{\prime}(u)$, where $\Delta$ is the discrete Laplacian. We mean with embedding that there exists a solution $q \in L^{\infty}(X)$ of $\Delta(q)=\gamma \cdot V^{\prime}(q)$ such that the configuration map $\phi: x \mapsto q\left(T^{n} x\right) \in \mathbf{R}^{\mathbf{Z}^{d}}$ is injective on a set $X^{\prime} \subset X$ of full measure. Such solutions exist for large $\gamma$ and are critical points of the functional $\mathcal{L}(q)=\int \sum_{i=1}^{n}\left(q\left(T_{i}\right)-q\right) / 2-V(q) d m$. The push-forward measure $\mu$ of $m$ on $\mathbf{R}^{\mathbf{Z}^{d}}$ has a compact support $Y$, and $\mu$-almost every $\phi(x)$ satisfies the partial difference 
equation. The map $\phi$ conjugates the $\mathbf{Z}^{d}$ systems $(X, T, m)$ and $(Y, S, \mu)$, where $S_{i}$ are the shifts on $\mathbf{R}^{\mathbf{Z}^{d}}$. The Hessian at a critical point $q$ is a discrete ergodic Laplacian $L=\Delta-V^{\prime \prime}(q)$ (see [Kni 94]).

Note added in proof. Since this paper was submitted (July 1994), the paper [Dua 94] appeared, which contains results overlapping with ours, obtained by different methods. The anti-integrable limit for coupled map lattices was discussed in the meantime in [Aub 95], as well as in [Jak 95].

\section{REFERENCES}

[Ang 90] S. Angenent. Monotone recurrence relations, their Birkhoff orbits and topological entropy. Erg. Th. Dyn. Sys., 10:15-41, 1990. MR 91b:58181

[Ang 92] S. Angenent. A remark on the topological entropy and invariant circles of an area preserving twist map. In "Twist Mappings and Their Applications", IMA Volumes in Mathematics, Vol. 44., Eds. R. Mc Gehee, K. Meyer, Springer, 1992, pp. 1-5. MR 94d:58078

[Aub 90] S. Aubry, G. Abramovici. Chaotic trajectories in the standard map. The concept of anti-integrability. Physica D, 43:199-219, 1990. MR 91j:58100

[Aub 92a] S. Aubry. The concept of anti-integrability: definition, theorems and applications to the standard map. In "Twist Mappings and Their Applications", IMA Volumes in Mathematics, Vol. 44., Eds. R. Mc Gehee, K. Meyer, Springer, 1992, pp. 7-54. MR 94c:58179

[Aub 92b] S. Aubry, R. S. Mackay, C. Baesens C. Equivalence of uniform hyperbolicity for symplectic twist maps and phonon gap for Frenkel-Kontorova models. Physica D, 56:123134, 1992. MR 93e:58144

[Aub 95] S. Aubry. Anti-integrability in dynamical and variational problems, Physica D 86:284296, 1995. CMP 1996:2. [Added in Proof]

[Ban 88] V. Bangert. Mather sets for twist maps and geodesics on tori. Dynamics Reported, 1:1-55, 1988. MR 90a:58145

[Bow 75] R. Bowen, D. Ruelle. The ergodic theory of axiom A flows. Inventiones Math., 29:181202, 1975. MR 52:1786

[Bro 75] M. Brown, W. D. Neumann. Proof of the Poincaré-Birkhoff fixed point theorem. Michigan Math. J., 24:21-31, 1975. MR 56:6646

[Cyc 87] H. L. Cycon, R. G. Froese, W. Kirsch, B. Simon. Schrödinger Operators. Texts and Monographs in Physics, Springer, 1987. MR 88g:35003

[Den 76] M. Denker, C. Grillenberger, K. Sigmund. Ergodic Theory on Compact Spaces. Lecture Notes in Math., No. 527, Springer, 1976. MR 56:15879

[Dua 94] P. Duarte. Plenty of elliptic islands for the standard family of area preserving maps, Ann. Inst. H. Poincaré Anal. Non Linéaire 11:359-409, 1994. MR 95k:58115. [Added in Proof]

[Fat 89] A. Fathi. Expansiveness, hyperbolicity and Hausdorff dimension. Commun. Math. Phys., 126: 249-262, 1989. MR 90m:58159

[Fon 90] E. Fontich. Transversal homoclinic points of a class of conservative diffeomorphisms. J. Diff. Equ., 5, 87:1-27, 1990. MR 91j:58095

[Fri 89] S. Friedland, J. Milnor. Dynamical properties of plane polynomial automorphisms. Ergod. Th. \& Dynam. Sys. 9:67-99, 1989. MR 90f:58163

[Her 83] M. Herman. Sur les courbes invariantes par les difféomorphismes de l'anneau. Astérisque, 103-104, Société Mathématique de France, 1983. MR 85m:58062

[Jak 95] A. Jakobsen and O. Knill. Iteration of the coupled map lattice construction, Phys. Lett. A 205:179-183, 1995. CMP 1996:1. [Added in Proof]

[Kat 80] A. Katok. Lyapunov exponents, entropy, and periodic orbits for diffeomorphisms. Publ.Math. IHES, 51:137-172, 1980. MR 81i:28022

[Kat 82] A. Katok. Some remarks on Birkhoff and Mather twist map theorems. Ergod. Th. \& Dynam. Sys., 2:185-194, 1982. MR 84m:58041 
[Kni 93] O. Knill. Isospectral deformations of random Jacobi operators. Commun. Math. Phys., 151:403-426, 1993. MR 94j:58136

[Kni 94] O. Knill. Isospectral deformation of discrete random Laplacians. In "On Three Levels", Ed.: M.Fannes et al., Plenum Press, New York, 1994, pp. 312-330.

[Laz 84] V. F. Lazutkin, D. Ya. Terman. Percival variational principle for invariant measures and commensurate-incommensurate phase transitions in one dimensional chains. Commun. Math. Phys, 94:511-522, 1984. MR 86k:58072

[Man 87] R. Mané. Ergodic Theory and differentiable Dynamics. Ergebnisse der Mathematik und ihrer Grenzgebiete; Ser. 3, Bd. 8, New York, Springer, 1987. MR 88c:58040

[Mat 82] J. Mather. Existence of quasi-periodic orbits for twist homeomorphism of the annulus. Topology, 21:457-467, 1982. MR 84g:58084

[Mat 84] J. Mather. Amount of rotation about a point and the Morse index. Comm. Math. Phys., 94:141-153, 1984. MR 86c:58051

[Mos 73] J. Moser. Stable and Random Motion in Dynamical Systems. Princeton University Press, Princeton, 1973. MR 56:1355

[New 89] S. Newhouse. Continuity properties of entropy. Annals of Mathematics, 129:215-235, 1989. MR 90f:58108; 90m:58117

[Orn 70] D. S. Ornstein. Factors of Bernoulli shifts are Bernoulli shifts. Adv. Math., 5:349-364, 1970. MR 43:478b

[Per 80] I. C. Percival. Variational principle for invariant tori and cantori. Amer. Instit. of Physics Conf. Proc., 57:310-320, 1980. MR 82m:58040

[Pet 83] K. Petersen. Ergodic Theory. Cambridge Studies in Advanced Mathematics 2. Cambridge University Press, Cambridge, 1983. MR 87i:28002

[You 81] L-S. Young. On the prevalence of horseshoes. Trans. Amer. Math. Soc., 263:75-88, 1981. MR 82g:58070

Division of Physics, Mathematics and Astronomy, California Institute of Technology, 91125 Pasadena, CAlifornia

E-mail address: knill@cco.caltech.edu 